\title{
A Nomogram Model to Predict Malignant Cerebral Edema in Ischemic Stroke Patients Treated with Endovascular Thrombectomy: An Observational Study
}

This article was published in the following Dove Press journal:

Neuropsychiatric Disease and Treatment

Mingyang Du, $1,2, *$

Xianjun Huang, (iD ${ }^{3,4, *}$ Shun $\mathrm{Li}^{2}$ Lili Xu, ${ }^{2}$ Bin Yan, ' Yi Zhang, (D) ${ }^{4}$

Huaiming Wang, ${ }^{1,5,6}$

Xinfeng Liu ${ }^{1,4}$

'Department of Neurology, Jinling Clinical College of Nanjing Medical University, Nanjing 210002, Jiangsu Province, People's Republic of China; ${ }^{2}$ Department of Cerebrovascular Disease Treatment Center, Nanjing Brain Hospital Affiliated to Nanjing Medical

University, Nanjing 210002, Jiangsu Province, People's Republic of China; ${ }^{3}$ Department of Neurology, Yijishan Hospital, Wannan Medical College, Wuhu, Anhui Province, People's Republic of China; ${ }^{4}$ Department of Neurology, Jinling Hospital, Southern Medical University, Nanjing 210002, Jiangsu Province, People's Republic of China; ${ }^{5}$ Department of Neurology, Jinling Hospital, Medical School of Nanjing University, Nanjing 210002, Jiangsu Province, People's Republic of China; ${ }^{6}$ Department of Neurology, The 80th Group Army Hospital of People's Liberation Army, Weifang, Shandong Province, People's Republic of China

*These authors contributed equally to this work

Correspondence: Xinfeng Liu

Department of Neurology, Jinling Clinical College of Nanjing Medical University, 305\# East Zhongshan Road, Nanjing 210002, Jiangsu, People's Republic of China

Tel +86- I895 I 919349

Fax +86-25-8480I86

Email xfliu2@vip.163.com

Huaiming Wang

Department of Neurology, Jinling Hospital, Medical School of Nanjing University,

Nanjing 210002 , Jiangsu Province, People's

Republic of China

Tel +86- I8951919349

Fax +86-25-8480I86I

Email wanghm89@I63.com
Purpose: Malignant cerebral edema (MCE) in patients undergoing endovascular thrombectomy (EVT) is not uncommon and can reduce the benefit of EVT. We aimed to develop a nomogram model to predict the risk of MCE in ischemic stroke patients after EVT.

Patients and Methods: We retrospectively collected patients treated with EVT caused by anterior circulation large vessel occlusion stroke at two comprehensive stroke centers. MCE was defined as midline shift $>5 \mathrm{~mm}$ at the septum pellucidum or pineal gland with obliteration of the basal cisterns or the need for early decompressive hemicraniectomy. A multivariate logistic model was utilized to construct the best-fit nomogram model. The discrimination and calibration of the nomogram were estimated using the area under the receiver operating characteristic curve (AUC-ROC) and Hosmer-Lemeshow test.

Results: A total of 370 patients (mean age, 67.2 \pm 11.9 years; male, 56.8\%) were enrolled in the final analysis. Among them, 71 (19.2\%) patients experienced MCE after EVT treatment. After adjustment for potential confounders, age, baseline National Institutes of Health Stroke Scale score, collateral circulation, fast blood glucose level and recanalization were independent predictors of MCE and were incorporated into the nomogram. The AUC-ROC value of the nomogram was 0.805 (95\% confidence interval [CI]: 0.750-0.860). The HosmerLemeshow goodness-of-fit test showed good calibration of the nomogram ( $\mathrm{P}=0.681$ ).

Conclusion: The nomogram composed of age, baseline National Institutes of Health Stroke Scale score, blood glucose level, collateral circulation and recanalization may predict the probability of MCE in anterior circulation large vessel occlusion stroke patients treated with EVT.

Keywords: endovascular thrombectomy, edema, nomogram, stroke

\section{Introduction}

Randomized controlled trials have validated the efficacy and safety of endovascular thrombectomy (EVT) in patients with anterior circulation large vessel occlusion stroke (LVOS). ${ }^{1-5}$ Moreover, more patients could benefit from EVT based on imaging screening. ${ }^{6,7}$ However, approximately $45 \%$ of patients with LVOS are still unable to perform functional recovery after EVT. ${ }^{8,9}$ Recent studies have shown that malignant cerebral edema (MCE) after EVT is not uncommon and might reduce the benefit-risk ratio of EVT. ${ }^{10-12} \mathrm{MCE}$ is one of the most serious complications of acute ischemic stroke (AIS), and the mortality rate in MCE 
patients is nearly $80 \% .{ }^{13}$ Although treatments of MCE are limited, a pooled analysis of randomized-controlled trials shows that early decompressive hemicraniectomy could decrease mortality and increase the chance of a favorable functional outcome. ${ }^{14}$ Therefore, the early prediction of MCE might bring more benefits for patients with AIS.

A recent systematic review indicated that the major determinants for MCE after stroke are younger age, high National Institutes of Health Stroke Scale (NIHSS) score, larger parenchymal hypoattenuation and the degree of reperfusion. ${ }^{15}$ Additionally, several studies in patients with intravenous thrombolysis (IVT) show that baseline blood glucose, onset-to-treatment time (OTT) and hyperdense artery sign were independent predictors of MCE. ${ }^{16,17}$ However, data on the prediction of MCE in patients treated with EVT are relatively scarce. Hence, in this study, we aimed to develop a nomogram model based on demographic and clinical features to predict MCE in patients treated with EVT.

\section{Patients and Methods}

\section{Study Population}

We retrospectively collected the demographic, clinical and radiographic data of patients with anterior circulation LVOS who underwent EVT at two comprehensive stroke centers (Jinling Hospital between January 2014 and December 2018 and Yijishan Hospital between July 2014 and February 2019). Patients were enrolled if they fulfilled the following inclusion criteria: (1) age $\geq 18$ years; (2) time from stroke onset to puncture (OTP) $\leq 8$ hours; (3) baseline NIHSS score $\geq 6$, baseline Alberta Stroke Program Early CT score (ASPECT) $\geq 6$, and prestroke modified Rankin Scale (mRS) score $<2$; (4) the internal carotid artery (ICA) or proximal segment (M1 or M2) of the middle cerebral artery (MCA) occlusion confirmed by preoperative imaging. The treatment protocol and methods were published previously. ${ }^{9,10}$ We excluded patients with extensive subarachnoid hemorrhage and midline shift or decompressive hemicraniectomy because of postprocedural hemorrhagic transformation. The study was approved by the Ethical Committee of Jinling Hospital and Yijishan Hospital (2010NLY-018). Due to its retrospective nature; patient consent was waived. All procedures performed in studies involving human participants were in accordance with the 1964 Helsinki declaration and its later amendments or comparable ethical standards. The data of patients were stored securely and confidentially in Jingling and Yijishan Hospitals.

\section{Malignant Cerebral Edema and Clinical Characteristics}

According to the follow-up imaging within 3-5 days after EVT, MCE was defined as midline shift $>5 \mathrm{~mm}$ at the septum pellucidum or pineal gland with obliteration of the basal cisterns or the need for decompressive hemicraniectomy. ${ }^{11,18}$ All neuroimaging data were reviewed by 2 physicians who were blinded to the clinical data. In case of disagreement, consensus was sought by discussion with a third physician.

The baseline clinical data included demographics, medical history, baseline NIHSS and ASPECT score, the Trial of ORG 10,172 in Acute Stroke Treatment (TOAST) classification and laboratory measures. Procedural characteristics, including OTP, time from stroke onset to recanalization (OTR), occlusion site, types of the procedure, bridging and rescue treatments, were all recorded. Recanalization status after the procedure was evaluated based on the modified Thrombolysis in Cerebral Infarction (mTICI) grading system. Successful recanalization was defined as an mTICI score of $2 b$ or 3 . Collateral circulation was assessed according to retrograde contrast opacification of vessels within the occluded area. The collateral score was classified as follows: grade 0 was assigned if there was little or no significant reconstitution in the territory of the occluded vessel or less than one-third of the occluded territory, grade 1 was assigned if the collaterals reached less than two-thirds of the occluded territory, and grade 2 was assigned if the collateralization reached more than two-thirds of the territory or the proximal main stem. ${ }^{19}$

The functional outcomes were assessed with a 90-day $\mathrm{mRS}$ score, and a favorable functional outcome was defined as $\mathrm{mRS}$ score $\leq 2$.

\section{Statistical Analysis}

Continuous variables are presented as the mean \pm standard deviation (SD) or as the median (interquartile range, IQR). Categorical variables are presented as percentages. Continuous variables were analyzed using the MannWhitney U-test. Categorical variables were analyzed using the Chi-square test or Fisher's exact test as appropriate. To construct the nomogram, multivariate regression analysis with Akaike information criterion was applied to select the significant predictors of MCE using a backward- 
selection method that included variables with a $\mathrm{P}$ value $<$ 0.2 in the univariate analysis. The nomogram was established according to the significant predictors in the multivariate regression analysis. Regression coefficients and odds ratios (ORs) with two-sided 95\% confidence intervals (CIs) for each variable included in the model were finally calculated. The collinearity of variables was evaluated by variation inflation factors.

The discriminative ability of the new nomogram was assessed by the area under the receiver operating characteristic curve (AUC-ROC). In addition, bootstrapping was used for internal validation. ${ }^{20}$ Calibration of the nomogram was assessed by a calibration plot and HosmerLemeshow test. The calibration plot is a graphic showing the relationship between the observed outcome frequencies and the predicted probabilities. In a well-calibrated model, the predictions should fall on a 45-degree diagonal line. Statistical analyses were computed using $\mathrm{R}$ version 3.0 (R Foundation, Vienna, Austria) and SPSS 22.0 (IBM, Armonk, NY, USA). A two-tailed value of $\mathrm{P}<0.05$ was considered significant.

\section{Results}

In total, 370 patients (mean age, $67.2 \pm 11.9$ years; male, $56.8 \%$ ) were enrolled for the final analysis. The flow chart of the inclusion of the study population is displayed in Figure 1. Of the 370 patients treated with EVT, the median OTP was 247 minutes, and baseline NIHSS scores and ASPECT scores were 17.0 and 9.0, respectively. Among them, $313(84.6 \%)$ patients received stent-retriever-first thrombectomy, 18 (4.9\%) received aspiration-first, and 39 $(10.5 \%)$ received angioplasty or stent-first procedure. Successful recanalization was achieved in 272 (73.5\%) patients. During the follow-up at 3 months, 167 (45.1\%) reached a favorable functional outcome (mRS, 0-2), and the overall mortality was $21.9 \%$.

\section{Predictors of Malignant Cerebral Edema}

There were 71 (19.2\%) patients who experienced MCE after EVT treatment. Table 1 shows the detailed demographic and clinical characteristics according to patients with and without MCE. In the univariate analysis, patients with MCE had a higher baseline NIHSS score $(18.0$ vs $16.0, \mathrm{P}<0.001)$ and a lower baseline ASPECT score ( 8 vs $9, \mathrm{P}=0.033$ ). Furthermore, ICA-T occlusion $(52.1 \%$ vs $24.7 \%, \mathrm{P}<0.001)$ and a lower degree of collateral circulation (grade $0,42.3 \%$ vs $13.7 \%$; grade $1,43.7 \%$ vs $44.1 \%$; grade $2,14 \%$ vs $42.2 \%$, $\mathrm{P}<0.001)$ occurred more frequently in MCE patients.

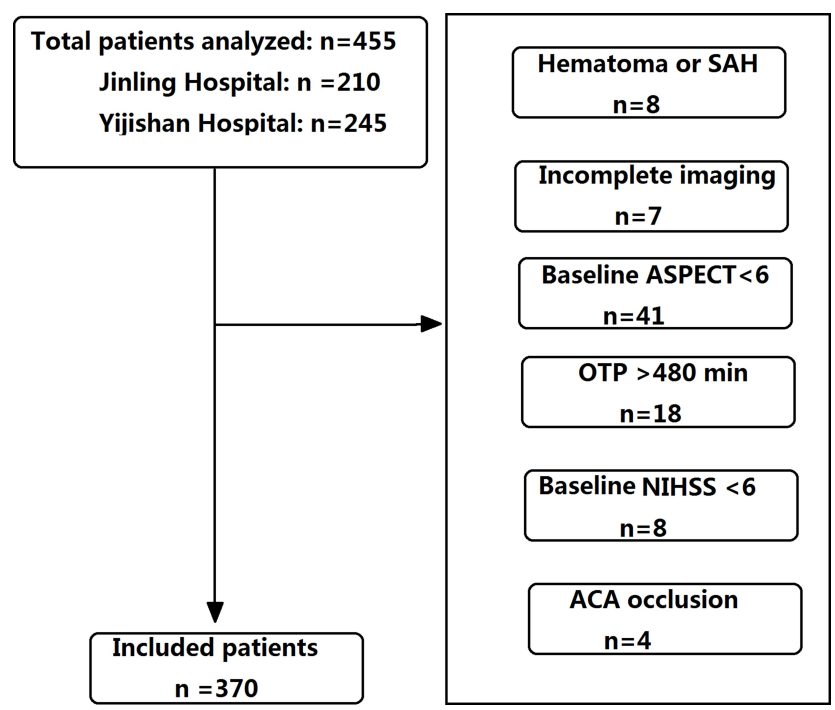

Figure I Flow chart of the inclusion of the study population. Abbreviations: ASPECT, Alberta Stroke Program Early CT; ACA, anterior cerebral artery; NIHSS, National Institutes of Health Stroke Scale; OTP, time from stroke onset to puncture; $\mathrm{SAH}$, subarachnoid hemorrhage.

Additionally, patients with MCE received a higher rate of rescue treatment $(28.2 \%$ vs $15.7 \%, \mathrm{P}=0.014)$ and spent a longer OTR (352 $\mathrm{min}$ vs $329 \mathrm{~min}, \mathrm{P}=0.015$ ). However, the rate of successful recanalization $(53.5 \%$ vs $78.3 \%, \mathrm{P}<$ 0.001) was lower in patients with MCE. Additionally, a significantly higher level of fast blood glucose $(9.1 \mathrm{mmol} /$ 1 vs $7.1 \mathrm{mmol} / \mathrm{l}, \mathrm{P}<0.001)$ and blood urea nitrogen (6.9 $\mathrm{mmol} / \mathrm{l}$ vs $6.2 \mathrm{mmol} / \mathrm{l}, \mathrm{P}=0.036$ ) was found in patients with MCE than in those without MCE.

After adjustment for potential confounders (variables with a $P$ value $<0.2$ in the univariate analysis), age (OR: 0.960, 95\% CI: 0.934-0.987, P = 0.004), baseline NIHSS score (OR: 1.076, 95\% CI: 1.016-1.140, $\mathrm{P}=0.013$ ), collateral circulation (grade 1 vs grade 0: OR: 0.360 , 95\% CI: $0.176-0.763, \mathrm{P}=0.005$, grade 2 vs grade 0 : OR: $0.127,95 \%$ CI: $0.051-0.315, \mathrm{P}<0.001)$, fast blood glucose level (OR: 1.180, 95\% CI: 1.086-1.281, P < $0.001)$ and recanalization status after the procedure (mTICI 2b-3 vs 0-2a, OR: 0.352, 95\% CI: 0.185-0.669, $\mathrm{P}=0.001)$ were independent predictors for $\mathrm{MCE}$ (Table 1). There was no significant statistical collinearity among the five independent predictors (Table S1).

\section{A Nomogram Model for the Prediction of Malignant Cerebral Edema}

The nomogram was developed by assigning a graphic initial score to each independent predictor with a point range from 0 to 100 , which was then summed to generate 
Table I Comparison of Variables Between All Patients with and without Malignant Cerebral Edema

\begin{tabular}{|c|c|c|c|c|c|c|c|}
\hline & Total & Non-MCE & MCE & \multirow[t]{2}{*}{$P$ value } & \multirow[t]{2}{*}{ OR } & \multirow[t]{2}{*}{$95 \% \mathrm{Cl}$} & \multirow[t]{2}{*}{$P$ value } \\
\hline & $n=370$ & $\mathrm{n}=\mathbf{2 9 9}$ & $n=71$ & & & & \\
\hline \multicolumn{8}{|l|}{ Demographics } \\
\hline Age, mean (SD) & $67.2(11.9)$ & $67.2(12.1)$ & $67.2(11.5)$ & 0.967 & 0.960 & $0.934-0.987$ & 0.004 \\
\hline Male sex, n (\%) & $210(56.8)$ & $174(58.2)$ & $36(50.7)$ & 0.252 & & & \\
\hline \multicolumn{8}{|l|}{ Medical history, n (\%) } \\
\hline Hypertension & $252(68.1)$ & $199(66.6)$ & $53(74.6)$ & 0.188 & & & \\
\hline Diabetes mellitus & $74(20.0)$ & $59(19.7)$ & $15(21.1)$ & 0.792 & & & \\
\hline Atrial fibrillation & $185(50.0)$ & $149(49.8)$ & $36(50.7)$ & 0.895 & & & \\
\hline TOAST, n (\%) & & & & 0.392 & & & \\
\hline LAA & $126(34.1)$ & $106(35.5)$ & $20(28.2)$ & & & & \\
\hline $\mathrm{CE}$ & $204(55.1)$ & $163(54.5)$ & $4 \mid(57.7)$ & & & & \\
\hline Others & $40(10.8)$ & $30(10.0)$ & $10(14.1)$ & & & & \\
\hline \multicolumn{8}{|l|}{ Baseline data } \\
\hline Baseline SBP, mean (SD) & $142.1(23.0)$ & $141.0(22.5)$ & $146.5(24.7)$ & 0.119 & & & \\
\hline Baseline DBP, mean (SD) & $81.9(14.2)$ & $81.3(13.7)$ & $84.4(16.1)$ & 0.134 & & & \\
\hline Baseline NIHSS, median (IQR) & $17(13-20)$ & $16(13-20)$ & $18(16-22)$ & $<0.001$ & 1.076 & $1.016-1.140$ & 0.013 \\
\hline Baseline ASPECT, median (IQR) & $9(8-10)$ & $9(8-10)$ & $8(8-10)$ & 0.033 & & & \\
\hline OTP, median (IQR) & $247(201-300)$ & $245(200-300)$ & $270(210-310)$ & 0.328 & & & \\
\hline OTR, median (IQR) & $333(277-390)$ & $329(277-382)$ & $352(290-420)$ & 0.015 & & & \\
\hline Occlusion site, $\mathbf{n}(\%)$ & & & & $<0.001$ & & & \\
\hline ICA* & $17(4.6)$ & $16(5.4)$ & I (I.4) & & & & \\
\hline ICA-T & III (30.0) & $74(24.7)$ & $37(52.1)$ & & & & \\
\hline MCA MI & $219(59.2)$ & $187(62.5)$ & $32(45.1)$ & & & & \\
\hline MCA M2 & $23(6.2)$ & $22(7.4)$ & I (1.4) & & & & \\
\hline Tandem, n (\%) & $64(17.3)$ & $42(14.0)$ & $12(16.9)$ & 0.540 & & & \\
\hline Collateral circulation, $\mathbf{n}(\%)$ & & & & $<0.001$ & & & \\
\hline Grade 0 & $71(19.2)$ & $41(13.7)$ & $30(42.3)$ & & & Reference & \\
\hline Grade I & $163(44.1)$ & $132(44.1)$ & $31(43.7)$ & & 0.360 & $0.176-0.736$ & 0.005 \\
\hline Grade 2 & $136(36.7)$ & $126(42.2)$ & $10(14.0)$ & & 0.127 & $0.051-0.315$ & $<0.001$ \\
\hline \multicolumn{8}{|l|}{ Laboratory test, mean (SD) } \\
\hline $\mathrm{FBG}, \mathrm{mmol} / \mathrm{l}$ & $7.5(3.3)$ & $7.1(3.0)$ & $9.1(4.0)$ & $<0.001$ & 1.180 & $1.086-1.281$ & $<0.001$ \\
\hline LDL, mmol/l & $2.4(0.9)$ & $2.4(0.8)$ & $2.4(1.3)$ & 0.993 & & & \\
\hline BUN, mmol/l & $6.3(2.5)$ & $6.2(2.2)$ & $6.9(3.6)$ & 0.036 & & & \\
\hline $\mathrm{Cr}, \mathrm{umol} / \mathrm{l}$ & $83.2(40.5)$ & $81.9(34.2)$ & $88.7(60.6)$ & 0.208 & & & \\
\hline \multicolumn{8}{|l|}{ Treatment, n (\%) } \\
\hline Bridging treatment & $86(23.2)$ & $65(21.7)$ & $21(29.6)$ & 0.160 & & & \\
\hline Type of procedure & & & & 0.153 & & & \\
\hline Stent retriever first & $313(84.6)$ & $249(83.3)$ & $64(90.2)$ & & & & \\
\hline Aspiration first & $18(4.9)$ & $14(4.7)$ & $4(5.6)$ & & & & \\
\hline Angioplasty or stent first & $39(10.5)$ & $36(12.0)$ & $3(4.2)$ & & & & \\
\hline Rescue treatment & $67(18.1)$ & $47(15.7)$ & $20(28.2)$ & 0.014 & & & \\
\hline $\mathrm{DHC}$ & $18(4.9)$ & $0(0.0)$ & $18(25.4)$ & $<0.001$ & & & \\
\hline \multicolumn{8}{|l|}{ Outcome, n (\%) } \\
\hline $\mathrm{mTICl}, 2 \mathrm{~b}-3$ & $272(73.5)$ & $234(78.3)$ & $38(53.5)$ & $<0.001$ & 0.352 & $0.185-0.669$ & 0.001 \\
\hline 90-day mRS (0-2) & $167(45.1)$ & $162(54.2)$ & $5(7.0)$ & $<0.001$ & & & \\
\hline 90-day mortality & $81(21.9)$ & $38(12.7)$ & $43(60.6)$ & $<0.001$ & & & \\
\hline
\end{tabular}

Note: *ICA occlusion between segments $\mathrm{CI}-\mathrm{C} 6$.

Abbreviations: MCE, malignant cerebral edema; TOAST, Trial of Org 10,172 in Acute Stroke Treatment; LAA, large-artery atherosclerosis; CE, cardio-embolism; SBP, systolic blood pressure; DBP, diastolic blood pressure; NIHSS, National Institutes of Health Stroke Scale; ASPECT, Alberta Stroke Program Early CT; OTP, symptom onset to groin puncture time; OTR, time from stroke onset to recanalization; ICA, internal carotid artery; MCA, middle cerebral artery; FBG, fast blood glucose; LDL, low-density lipoprotein; BUN, blood urea nitrogen; $\mathrm{Cr}$, creatinine; DHC, decompressive hemicraniectomy; mTICl, modified thrombolysis in cerebral infarction; mRS, modified Rankin Scale. 
a total score and converted into a percentage representing at $>12.0 \mathrm{mmol} / \mathrm{L}$, baseline NIHSS score at 20.0, $\mathrm{mTICI}$ an individual risk of the development of MCE after EVT. 2b-3, and grade 0 of collateral circulation would have The details of the new nomogram are shown in Figure 2. a total of 160.0 points (26.0 points for age, 42.0 points For example, a patient with age at 65 years, blood glucose for blood glucose, 32.0 points for baseline NIHSS score,

Points

Age

Baseline NIHSS Score

Fast blood glucose

Collateral circulation

$\mathrm{mTICl}$

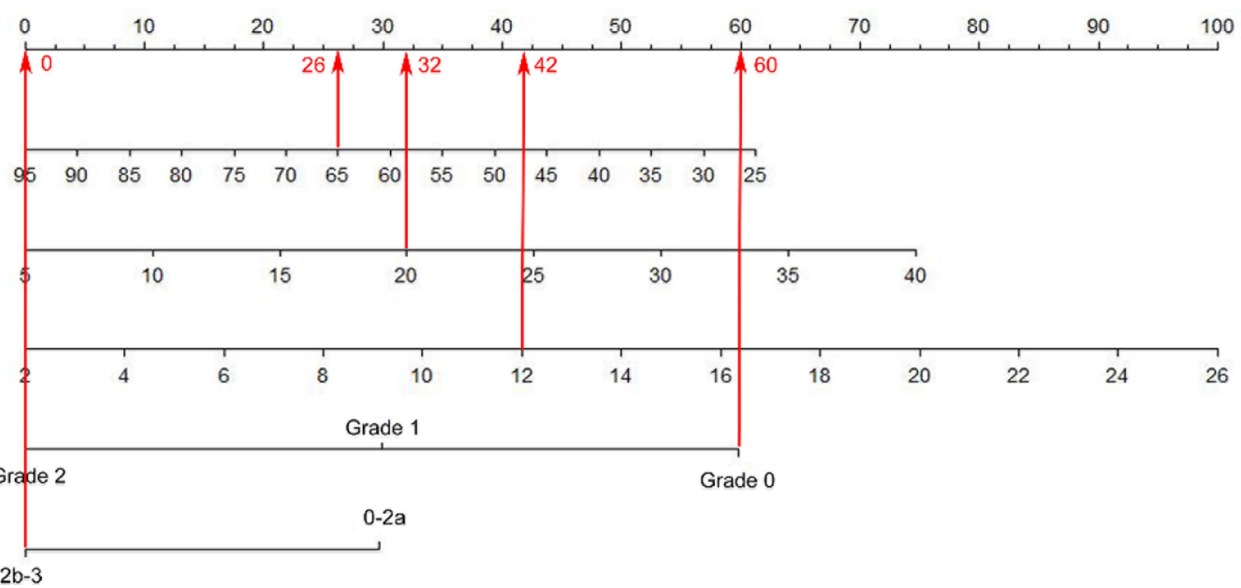

Total point

The risk of MCE

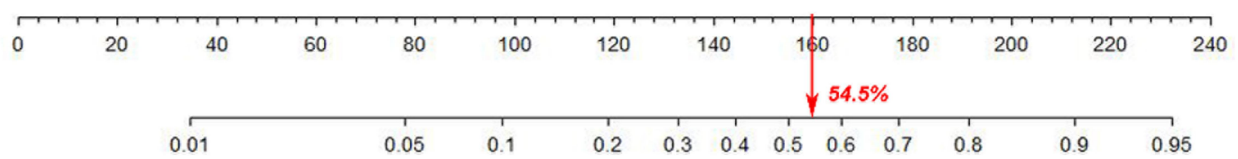

Figure 2 The nomogram model for predicting the probability of malignant cerebral edema in patients after thrombectomy. The variables, including age, baseline NIHSS score, FBG, the grade of collateral circulation and the degree of $\mathrm{TICl}$, could predict the risk of MCE in patients after thrombectomy. The vertical line of the "Points" axis determines the number of points of every variable for predicting the development of MCE. Add the number of points from each variable. Mark this sum on the "Total point" axis, and draw a vertical line down to meet the "The risk of MCE" axis, to find the patient' probability of developing MCE.

Abbreviations: $\mathrm{mTICl}$, modified Thrombolysis in Cerebral Infarction; MCE, malignant cerebral edema; NIHSS, National Institutes of Health Stroke Scale.

A

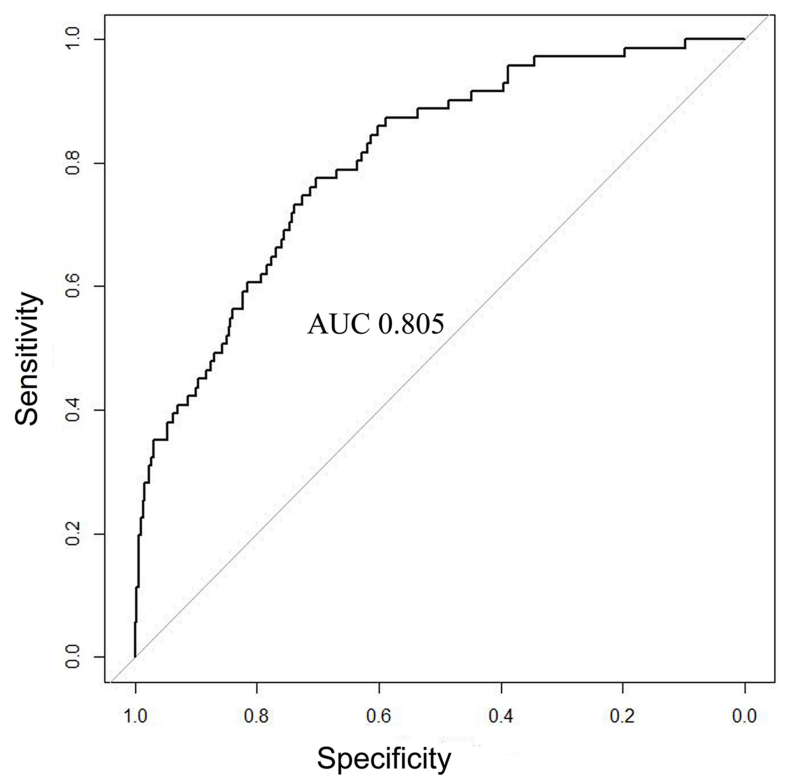

B

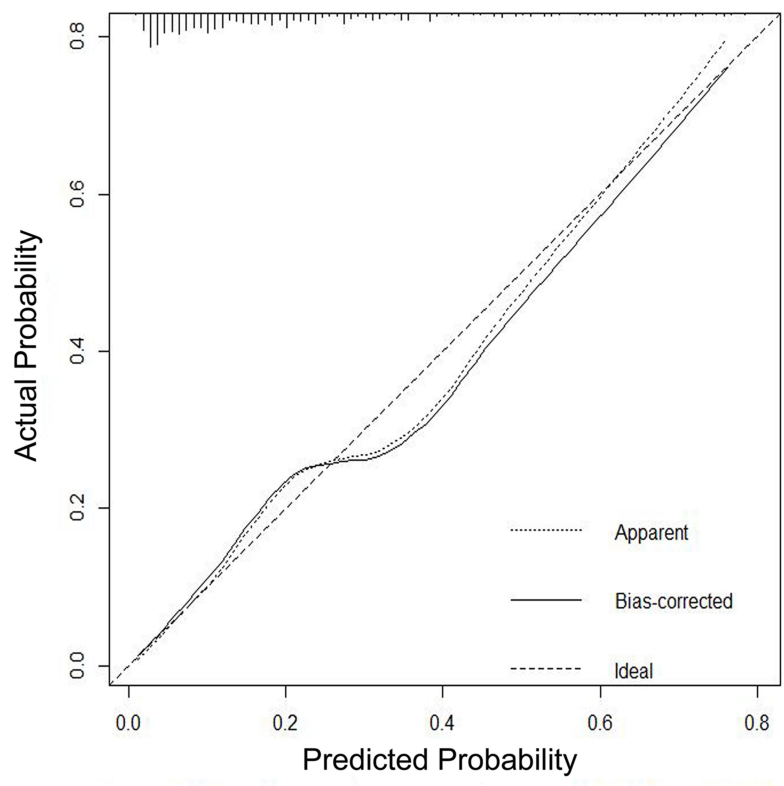

$B=1000$ repetitions, boot

Mean absolute error $=0.019, \mathrm{n}=370$

Figure 3 The receiver operating characteristic curve $(\mathbf{A})$ and the calibration plot $(\mathbf{B})$ of the nomogram model. The dashed line is the reference line where an ideal nomogram would lie. The dotted line is the performance of the nomogram, while the solid line corrects for any bias in the nomogram. 


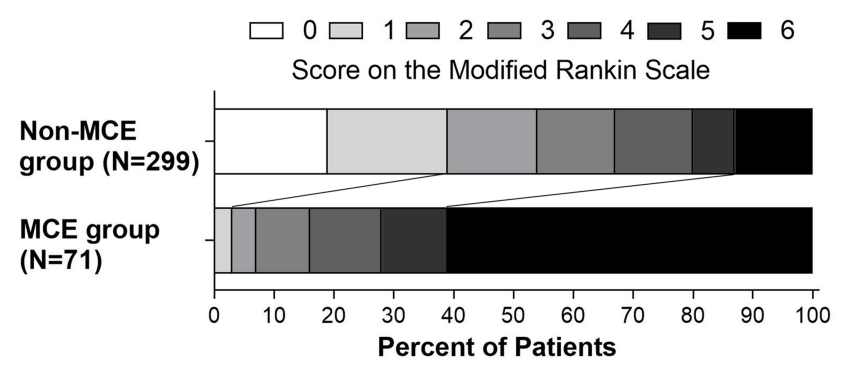

Figure 4 Distribution of modified Rankin scale (mRS) scores at 90 days according to the presence of malignant cerebral edema.

0 points for mTICI $2 \mathrm{~b}-3$, and 60.0 points for collateral circulation). The predicted MCE was approximately $54.5 \%$ for this patient.

The discrimination of the nomogram was measured by calculating the AUC-ROC value, which was 0.805 (95\% CI: $0.750-0.860$, Figure 3A). A generally accepted approach suggests that an AUC value of more than 0.75 reflects clearly useful discrimination. ${ }^{21}$ Also, the ROC curve analysis demonstrated the optimal cutoff value of total nomogram score as a MCE indicator was estimated to be 115.0, which yielded a sensitivity of $77.5 \%$ and a specificity of $70.2 \%$. The model was internally validated using 1000 bootstrap samples to calculate the discrimination with accuracy, and the good predictive performance of the nomogram was also confirmed, yielding a notable AUC of 0.805. The HosmerLemeshow goodness-of-fit test showed good calibration of the nomogram $(\mathrm{P}=0.681)$. Additionally, the calibration plot for the nomogram model showed adequate agreement between predictors calculated with the nomogram and actual MCE risk (Figure 3B).

\section{Clinical Outcomes of Malignant Cerebral Edema}

The results of univariate analysis of the 90-day favorable outcome are reported in Table $\mathrm{S} 2$. In the multivariate analysis, the presence of MCE was the major predictor of poor outcome after EVT treatment (OR: 13.489, 95\% CI: 3.322-54.771, $\mathrm{P}<0.001)$. We also found that patients with MCE had an increased $\mathrm{mRS}$ score $(\mathrm{P}<0.001$, Figure 4$)$.

\section{Discussion}

In the present study, we found that the occurrence of $\mathrm{MCE}$ after EVT was an independent risk factor for poor clinical outcome at 90 days. ${ }^{16}$ Moreover, our study suggested that several clinical factors, including age, baseline NIHSS score, collateral circulation, FBG, and recanalization status after the procedure, were significantly associated with the development of MCE.

Consistent with the results of previous studies, our findings showed that a higher NIHSS score was an independent predictor for MCE after EVT. The severity of the neurological deficit on admission assessed by the NIHSS score has been suggested to be a stronger predictor of stroke outcome. Moreover, previous studies showed that a higher NIHSS score was associated with cerebral edema after stroke. ${ }^{15,17}$ Additionally, a higher NIHSS score may imply poor collateral status and a larger infarct core, which are important risk factors for MCE. ${ }^{22,23}$

Recanalization is strongly associated with improved clinical outcomes after EVT. Our study showed that successful recanalization could significantly decrease the development of MCE. Although abrupt reperfusion is associated with secondary brain damage in animal models of ischemic stroke, ${ }^{24,25}$ recent studies have confirmed that there is no significant lesion growth after successful recanalization in patients treated with EVT. ${ }^{26}$ Furthermore, cerebral edema reduction could increase the clinical benefit of thrombectomy in AIS patients. ${ }^{11,27}$

In the present study, we found that FBG was a powerful predictor of MCE after EVT. Several studies have shown that hyperglycemia could affect clinical outcomes after EVT. ${ }^{28,29}$ However, the exact mechanism is still unclear. One of the possible mechanisms is that hyperglycemia may increase matrix metalloproteinase- 9 activity and disrupt the blood-brain barrier, which may also lead to the occurrence of MCE in patients with AIS. ${ }^{30}$

Notably, we did not find a significant difference in age between the MCE and non-MCE groups in the univariate analysis. However, since a recent meta-analysis showed that age is a reliable predictor of MCE in ischemic stroke, ${ }^{15}$ we still included age in the regression analysis in the current study. Unexpectedly, we found that old age was a protective factor for MCE after EVT, which was consistent with the results in the previous meta-analysis. We speculated that the possible reason was that age-related brain atrophy may provide buffering space for brain swelling.

An accurate estimate of the development of MCE after EVT may provide important information to clinicians in clinical decisions and prognostic assessments. Hence, in previous studies, several scoring systems were established to predict the development of MCE in patients with AIS, including those treated with IVT. ${ }^{18,31,32}$ However, there is no suitable scoring system for patients treated with EVT. 
To our knowledge, the present study may be the first attempt to develop a nomogram model for predicting the development of MCE after EVT.

Nomograms are important tools for modern medical decision-making. Compared with previous scoring systems, such as the EDEMA score and DASH score, ${ }^{18,31}$ the nomogram model can assign a probability of developing MCE after EVT, which often provides better-individualized assessments that facilitate management-related decisions. Additionally, the nomogram has higher accuracy and better discrimination ability and is more convenient to use. In the present study, the nomogram model incorporated with five easily available variables had a favorable discrimination ability, as supported by the AUC-ROC value of 0.805 and the optimal calibration curves. Thus, the nomogram model can facilitate the early identification of patients who may develop MCE and support rational decision-making for early DHC of patients with a very high probability of MCE after EVT.

In the present study, we first provided a nomogram model to predict MCE after EVT. However, our study has some limitations. First, this nomogram was based on retrospective data from only 2 institutions. Missing data may affect the accuracy of the model. Second, this study only included a subset of patients who were eligible for EVT according to current guidelines, which may lead to selection bias. Also, the model cannot be generalized to other patient cohorts. Third, known neuroradiologic predictors, such as infarct core, were not available in our study. ${ }^{23}$ Future studies are warranted to investigate whether the integration of novel markers would help improve the accuracy of nomogram prediction.

The nomogram composed of age, baseline NIHSS score, collateral circulation, fast blood glucose level, and recanalization status may predict the risk of MCE in ischemic stroke patients after EVT treatment. Future studies are warranted to externally validate our nomogram in different populations.

\section{Acknowledgments}

The project is supported by the National Natural Science Foundation of China (No.81870946, 81530038), National Key Research and Development Program (No. 2017YFC1307901), Natural Science Foundation of Jiangsu Province (No. BE2016748), and Outstanding Talents Program of Jiangsu Province (No. JCRCB2016005).

\section{Disclosure}

The authors have no conflicts of interest.

\section{References}

1. Berkhemer OA, Fransen PS, Beumer D, et al. A randomized trial of intraarterial treatment for acute ischemic stroke. $N$ Engl $J$ Med. 2015;372(1):11-20. doi:10.1056/NEJMoa1411587

2. Campbell BC, Mitchell PJ, Kleinig TJ, et al. Endovascular therapy for ischemic stroke with perfusion-imaging selection. $N$ Engl J Med. 2015;372(11):1009-1018. doi:10.1056/NEJMoa1414792

3. Goyal M, Demchuk AM, Menon BK, et al. Randomized assessment of rapid endovascular treatment of ischemic stroke. $N$ Engl J Med. 2015;372(11):1019-1030.

4. Jovin TG, Chamorro A, Cobo E, et al. Thrombectomy within 8 hours after symptom onset in ischemic stroke. $N$ Engl J Med. 2015;372 (24):2296-2306. doi:10.1056/NEJMoa 1503780

5. Saver JL, Goyal M, Bonafe A, et al. Stent-retriever thrombectomy after intravenous t-PA vs. t-PA alone in stroke. $N$ Engl $J$ Med. 2015;372(24):2285-2295. doi:10.1056/NEJMoa1415061

6. Albers GW, Marks MP, Kemp S, et al. Thrombectomy for stroke at 6 to 16 hours with selection by perfusion imaging. $N$ Engl $J$ Med. 2018;378(8):708-718. doi:10.1056/NEJMoa1713973

7. Nogueira RG, Jadhav AP, Haussen DC, et al. Thrombectomy 6 to 24 hours after stroke with a mismatch between deficit and infarct. N Engl J Med. 2018;378(1):11-21. doi:10.1056/NEJMoa1706442

8. Goyal M, Menon BK, van Zwam WH, et al. Endovascular thrombectomy after large-vessel ischaemic stroke: a meta-analysis of individual patient data from five randomised trials. Lancet. 2016;387 (10029):1723-1731. doi:10.1016/S0140-6736(16)00163-X

9. Zi W, Wang H, Yang D, et al. Clinical effectiveness and safety outcomes of endovascular treatment for acute anterior circulation ischemic stroke in China. Cerebrovasc Dis. 2017;44(5-6):248-258.

10. Huang X, Yang Q, Shi X, et al. Predictors of malignant brain edema after mechanical thrombectomy for acute ischemic stroke. J Neurointerv Surg. 2019;11(10):994-998. doi:10.1136/neurintsurg2018-014650

11. Kimberly WT, Dutra BG, Boers AMM, et al. Association of reperfusion with brain edema in patients with acute ischemic stroke: a secondary analysis of the mr clean trial. JAMA Neurol. 2018;75 (4):453-461. doi:10.1001/jamaneurol.2017.5162

12. Davoli A, Motta C, Koch G, et al. Pretreatment predictors of malignant evolution in patients with ischemic stroke undergoing mechanical thrombectomy. J Neurointerv Surg. 2018;10(4):340-344. doi:10.1136/neurintsurg-2017-013224

13. Huttner HB, Schwab S. Malignant middle cerebral artery infarction: clinical characteristics, treatment strategies, and future perspectives. Lancet Neurol. 2009;8(10):949-958. doi:10.1016/S1474-4422(09) 70224-8

14. Vahedi K, Hofmeijer J, Juettler E, et al. Early decompressive surgery in malignant infarction of the middle cerebral artery: a pooled analysis of three randomised controlled trials. Lancet Neurol. 2007;6 (3):215-222. doi:10.1016/S1474-4422(07)70036-4

15. Wu S, Yuan R, Wang Y, et al. Early prediction of malignant brain edema after ischemic stroke. Stroke. 2018;49(12):2918-2927. doi:10.1161/STROKEAHA.118.022001

16. Strbian D, Meretoja A, Putaala J, Kaste M, Tatlisumak T, Helsinki Stroke Thrombolysis Registry G. Cerebral edema in acute ischemic stroke patients treated with intravenous thrombolysis. Int J Stroke. 2013;8(7):529-534. doi:10.1111/j.1747-4949.2012.00781.x

17. Thoren M, Azevedo E, Dawson J, et al. Predictors for cerebral edema in acute ischemic stroke treated with intravenous thrombolysis. Stroke. 2017;48(9):2464-2471. doi:10.1161/STROKEAHA.117.018 223

18. Ong CJ, Gluckstein J, Laurido-Soto O, Yan Y, Dhar R, Lee JM. Enhanced detection of edema in malignant anterior circulation stroke (EDEMA) score: a risk prediction tool. Stroke. 2017;48 (7):1969-1972. doi:10.1161/STROKEAHA.117.016733 
19. Christoforidis GA, Mohammad Y, Kehagias D, Avutu B, Slivka AP. Angiographic assessment of pial collaterals as a prognostic indicator following intra-arterial thrombolysis for acute ischemic stroke. AJNR Am J Neuroradiol. 2005;26(7):1789-1797.

20. Kim Y, Margonis GA, Prescott JD, et al. Nomograms to predict recurrence-free and overall survival after curative resection of adrenocortical carcinoma. JAMA Surg. 2016;151(4):365-373.

21. D'Agostino R, Nam B. Evaluation of the performance of survival analysis models: Discrimination and calibration measures. In: Balakrishnan N, Rao CR, Eds. Handbook of statistics v23: Advances in survival analysis. Amsterdam, the Netherlands: Elsevier; 2004.

22. Dohmen C, Galldiks N, Bosche B, Kracht L, Graf R. The severity of ischemia determines and predicts malignant brain edema in patients with large middle cerebral artery infarction. Cerebrovasc Dis. 2012;33(1):1-7. doi:10.1159/000330648

23. Thomalla G, Hartmann F, Juettler E, et al. Prediction of malignant middle cerebral artery infarction by magnetic resonance imaging within 6 hours of symptom onset: A prospective multicenter observational study. Ann Neurol. 2010;68(4):435-445. doi:10.1002/ ana. 22125

24. Kidwell CS, Saver JL, Starkman S, et al. Late secondary ischemic injury in patients receiving intraarterial thrombolysis. Ann Neurol. 2002;52(6):698-703. doi:10.1002/ana.10380

25. Li F, Silva MD, Liu KF, et al. Secondary decline in apparent diffusion coefficient and neurological outcomes after a short period of focal brain ischemia in rats. Ann Neurol. 2000;48(2):236-244. doi:10.1002/ 1531-8249(200008)48:2<236::AID-ANA14>3.0.CO;2-7
26. Gauberti M, Lapergue B, Martinez de Lizarrondo S, et al. Ischemiareperfusion injury after endovascular thrombectomy for ischemic stroke. Stroke. 2018;49(12):3071-3074. doi:10.1161/STROKEAHA.118.022015

27. Broocks G, Hanning U, Flottmann F, et al. Clinical benefit of thrombectomy in stroke patients with low ASPECTS is mediated by oedema reduction. Brain. 2019;142(5):1399-1407. doi:10.1093/ brain/awz057

28. Chen X, Liu Z, Miao J, et al. High stress hyperglycemia ratio predicts poor outcome after mechanical thrombectomy for ischemic stroke. J Stroke Cerebrovasc Dis. 2019;28(6):1668-1673. doi:10.1016/j. jstrokecerebrovasdis.2019.02.022

29. Kim JT, Liebeskind DS, Jahan R, et al. Impact of hyperglycemia according to the collateral status on outcomes in mechanical thrombectomy. Stroke. 2018;49(11):2706-2714. doi:10.1161/ STROKEAHA.118.022167

30. Serena J, Blanco M, Castellanos M, et al. The prediction of malignant cerebral infarction by molecular brain barrier disruption markers. Stroke. 2005;36(9):1921-1926. doi:10.1161/01.STR.0000177870.14967.94

31. Battey TW, Karki M, Singhal AB, et al. Brain edema predicts outcome after nonlacunar ischemic stroke. Stroke. 2014;45 (12):3643-3648. doi:10.1161/STROKEAHA.114.006884

32. Shimoyama T, Kimura K, Uemura J, et al. The DASH score: a simple score to assess risk for development of malignant middle cerebral artery infarction. J Neurol Sci. 2014;338(1-2):102-106. doi:10.1016/ j.jns.2013.12.024
Neuropsychiatric Disease and Treatment

\section{Publish your work in this journal}

Neuropsychiatric Disease and Treatment is an international, peerreviewed journal of clinical therapeutics and pharmacology focusing on concise rapid reporting of clinical or pre-clinical studies on a range of neuropsychiatric and neurological disorders. This journal is indexed on PubMed Central, the 'PsycINFO' database and CAS, and

\section{Dovepress}

is the official journal of The International Neuropsychiatric Association (INA). The manuscript management system is completely online and includes a very quick and fair peer-review system, which is all easy to use. Visit http://www.dovepress.com/testimonials.php to read real quotes from published authors. 